Article

\title{
Dielectric Spectroscopy of Water Dynamics in Functionalized UiO-66 Metal-Organic Frameworks
}

\author{
Sergejus Balčiūnas ${ }^{1}$, Diana Pavlovaitè ${ }^{1}$, Martynas Kinka ${ }^{1}$, Jyun-Yi Yeh ${ }^{2}$, Po-Chun Han ${ }^{2}$, \\ Fa-Kuen Shieh ${ }^{3}$, Kevin C.-W. Wu ${ }^{2}$, Mantas Šimènas ${ }^{1}{ }^{1}$, Robertas Grigalaitis ${ }^{1}$ and \\ Jūras Banys ${ }^{1, *}$ \\ 1 Faculty of Physics, Vilnius University, Sauletekio av. 9, LT-10222 Vilnius, Lithuania; \\ sergejus.balciunas@ff.vu.lt (S.B.); diana.pavlovaite@ff.stud.vu.lt (D.P.); martynas.kinka@ff.vu.lt (M.K.); \\ mantas.simenas@ff.vu.lt (M.Š.); robertas.grigalaitis@ff.vu.lt (R.G.) \\ 2 Department of Chemical Engineering, National Taiwan University, Taipei 10617, Taiwan; \\ d07551002@ntu.edu.tw (J.-Y.Y.); a242520002000@gmail.com (P.-C.H.); kevinwu@ntu.edu.tw (K.C.-W.W.) \\ 3 Department of Chemistry, National Central University, Chung-Li 32001, Taiwan; fshieh@cc.ncu.edu.tw \\ * Correspondence: juras.banys@ff.vu.lt
}

Received: 21 March 2020; Accepted: 20 April 2020; Published: 23 April 2020

\begin{abstract}
We present a dielectric spectroscopy study of dipolar dynamics in the hydrated UiO-66(Zr) type metal-organic frameworks (MOFs) functionalized with $-\mathrm{NH}_{2}$ and $-\mathrm{F}$ groups. Experiments are performed in a broad temperature and frequency ranges allowing us to probe several dipolar relaxations. For both samples at temperature below $220 \mathrm{~K}$, we observe confined supercooled water dynamics, which can be described by the Arrhenius law. At slightly higher temperature, a second less pronounced dipolar relaxation is identified, and its origin is discussed. At even higher temperature, the dielectric permittivity exhibits anomalous increase with increasing temperature due to the proton conductivity. Upon further heating, the permittivity shows a sudden decrease indicating a reversible removal of water molecules. Measurements of the dehydrated samples reveal absence of all three dipolar processes.
\end{abstract}

Keywords: metal-organic framework; UiO-66; dielectric spectroscopy; water dynamics

\section{Introduction}

Metal-organic frameworks (MOFs) are crystalline compounds consisting of metal centers joined together by organic linkers into highly porous structures [1]. A vast diversity of these building units allows synthesis of various MOF structures with different topologies and functionalities [2]. The high porosity and tunability of these compounds is expected to be utilized for selective gas adsorption [3,4], separation [5], and storage [6,7] as well as chemical catalysis [8], drug delivery [9] and other applications [10].

A highly promising family of such compounds is $\mathrm{UiO}$ MOFs (UiO stands for University of Oslo), which exhibit superior thermal and chemical stability as well as high porosity [11]. One of the most popular members of these compounds is UiO-66( $\mathrm{Zr})$ MOF consisting of $\mathrm{Zr}_{6} \mathrm{O}_{4}(\mathrm{OH})_{4}$ secondary building units (SBUs), which are connected by 1,4-benzene-dicarboxylate (BDC) linkers into porous structures with $F m \overline{3} m$ face-centered cubic structure (see Figure 1) [11]. The framework has octahedral pores connected to smaller tetrahedral cavities via relatively narrow windows of about $5 \AA$ diameter. The pronounced stability of UiO-66 stems from the robust SBUs and their high coordination number, which results in the exceptional defect tolerance [12,13]. 


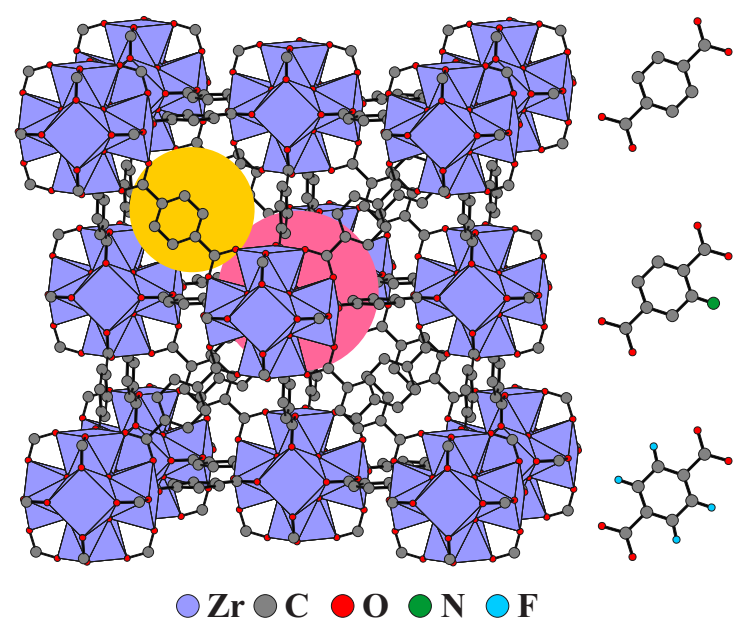

Figure 1. Crystal structure of UiO-66 (left). Examples of octahedral and tetrahedral pores are indicated in pink and yellow, respectively. BDC linker and its functionalized $\mathrm{BDC}-\mathrm{NH}_{2}$ and $\mathrm{BDC}-\mathrm{F}_{4}$ analogues (right). Structural data taken from Ref. [11]. Hydrogen atoms of BDC linkers are not shown for clarity.

The gas adsorption, gas separation as well as other physical and chemical properties of UiO-66 MOF can be significantly modified by introducing functionalized BDC linkers with various chemical groups (e.g., $-\mathrm{NH}_{2},-\mathrm{OH},-\mathrm{Br},-\mathrm{COOH},-\mathrm{NO}_{2}$ and $-\mathrm{F}$ ), while retaining the stability of the framework [14-17]. For example, functionalization by $-\mathrm{NH}_{2}$ group results in a 20-fold increase in phosphate-ester hydrolysis rate [18], increased capacity to detect explosive simulants [19], better mechanical stability [20] and significant improvement of $\mathrm{CO}_{2} / \mathrm{CH}_{4}$ separation performance [21,22] compared with pristine UiO-66 MOF.

Attachment of the functional groups is also expected to influences the linker dynamics in UiO MOFs. In addition to NMR spectroscopy [23,24] and quasi-elastic neutron scattering [25], a method of choice to study such dynamic effects of polar linkers and guest molecules is dielectric spectroscopy [26-29]. This technique was used to probe the linker dynamics of functionalized UiO-66 with polar $-\mathrm{Br},-\mathrm{OH}$ and $-\mathrm{NH}_{2}$ groups [30]. In addition, the same method was also employed to study dynamics of the biologically active guest molecules such ibuprofen and caffeine adsorbed in UiO-66- $\mathrm{NH}_{2} \mathrm{MOF}[31,32]$. The dielectric spectroscopy also revealed water dynamics and enhancement of proton conductivity in UiO-66 with acidic $-\mathrm{COOH}$ polar groups [33]. Two dipolar relaxation processes on different time scales were observed and assigned to dynamics of the confined water clusters and cooperative motion of the water molecules bound to the pore wall.

Despite these investigations, the dynamic and sorption properties of water in other functionalized analogues of UiO-66 are poorly studied, though elucidation of such processes is essential for applications of these and other MOFs for $\mathrm{CO}_{2}$ adsorption and separation [4]. Thus, in this work, we use dielectric spectroscopy to probe the dipolar dynamics in the hydrated functionalized $\mathrm{UiO}-66-\mathrm{NH}_{2}$ and UiO-66- $\mathrm{F}_{4} \mathrm{MOFs}$ in a broad temperature and frequency ranges. Both functional groups are expected to exhibit different degree of hydrophobicity motivating their choice for this study. We reveal three different dynamic processes of water, which are related to the supercooled confined water and proton conductivity.

\section{Results and Discussion}

First we performed temperature dependent dielectric spectroscopy experiments of UiO-66- $\mathrm{NH}_{2}$ and UiO-66- $\mathrm{F}_{4}$ powder samples starting from the hydrated forms. Before the measurements, samples were kept in a high humidity atmosphere for $24 \mathrm{~h}$ to achieve maximum hydration level. The measurement cycle started by cooling from the room temperature to about $125 \mathrm{~K}$ at ambient atmosphere followed by heating to above $400 \mathrm{~K}$. 
The temperature dependences of the real $\varepsilon^{\prime}$ and imaginary $\varepsilon^{\prime \prime}$ parts of the complex dielectric permittivity $\varepsilon^{*}=\varepsilon^{\prime}-i \varepsilon^{\prime \prime}$ of hydrated $\mathrm{UiO}-66-\mathrm{NH}_{2}$ and $\mathrm{UiO}-66-\mathrm{F}_{4}$ samples on heating are presented in Figure 2. Both samples reveal three frequency dependent processes. The weakest dispersive process P1 is visible below $220 \mathrm{~K}$. With increasing temperature, the second process P2 enters our measurement frequency window in the 220-250 K temperature region and is superimposed by the strong response of the third process $\mathrm{P} 3$ at higher temperature.
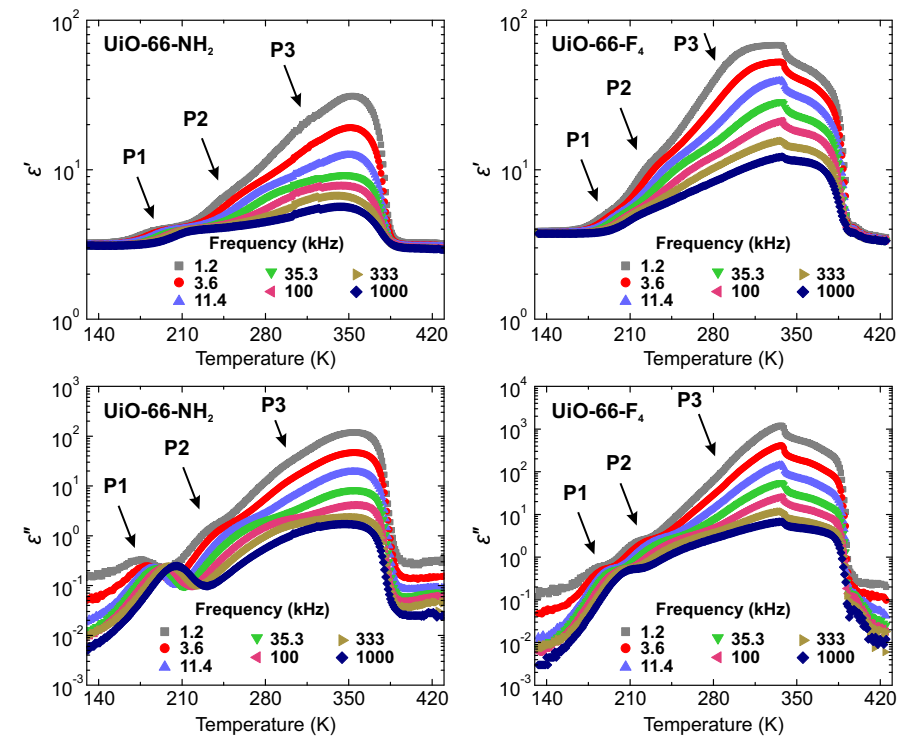

Figure 2. Temperature dependence of the complex dielectric permittivity of $\mathrm{UiO}-66-\mathrm{NH}_{2}$ and $\mathrm{UiO}-66-\mathrm{F}_{4}$ hydrated powder samples at several selected frequencies. Three processes, P1, P2 and P3, related to the adsorbed water are indicated by the arrows. Measurements performed on heating.

The real and imaginary parts of $\varepsilon^{*}$ start to decrease at about $350 \mathrm{~K}$ indicating dehydration of the samples [34]. This result is supported by the measurements of the temperature dependent weight loss of hydrated samples (Figure S3), where a significant dehydration continues up to about $370 \mathrm{~K}$. At slightly higher temperature the dielectric permittivity exhibits a sudden drop. A much smaller decrease of $\varepsilon^{*}$ is also observed at about $330 \mathrm{~K}$ for UiO-66- $\mathrm{F}_{4}$ sample, though its origin is unclear. Subsequently, after the dehydration nitrogen gas was applied and another cooling was performed under nitrogen atmosphere. The obtained flat responses on cooling (Figure 3) indicate that all three processes originate from the adsorbed water molecules within the pores or on the crystallite surfaces. Application of $\mathrm{N}_{2}$ gas during the measurements effectively prevents sample rehydration, which usually takes place in MOFs if cooled under ambient air conditions [28,35].

The three processes observed in the hydrated MOFs were further studied by analyzing the temperature dependences of the dielectric data at different frequencies. The frequency dependence of $\varepsilon^{*}$ is presented in Figures S4 and S5 revealing lack of clearly distinguishable relaxations. Since the dielectric response in our case is likely related to proton transport, we have used the electric modulus $M^{*}$ representation [36,37]. Generally, for a dielectric relaxation process, a relaxation peak appears in both $M^{*}$ and $\varepsilon^{*}$ representations, while for a pure conduction process the peak would be only seen in the $M^{*}$ spectrum. It was demonstrated that the absence of the peak in the imaginary part of $\varepsilon^{*}$ versus frequency shows a long-range conductivity phenomenon, while its presence reveals localized dynamics [38]. In our case, $M^{*}$ representation provides a much better separation of overlapping processes and allows to calculate the main relaxation parameters. The complex electric modulus $M^{*}$ is defined as the inverse of the complex dielectric permittivity $\varepsilon^{*}$ :

$$
M^{*}=\frac{1}{\varepsilon^{*}}=\frac{\varepsilon^{\prime}}{\varepsilon^{\prime 2}+\varepsilon^{\prime \prime 2}}+i \frac{\varepsilon^{\prime \prime}}{\varepsilon^{\prime 2}+\varepsilon^{\prime \prime 2}}=M^{\prime}+i M^{\prime \prime},
$$


where $M^{\prime}$ and $M^{\prime \prime}$ denote the real and imaginary parts of $M^{*}$, respectively. In our case, all three processes appeared as separated peaks in the frequency dependence of $M^{\prime \prime}$ (Figure 4) demonstrating a clear relaxation character.

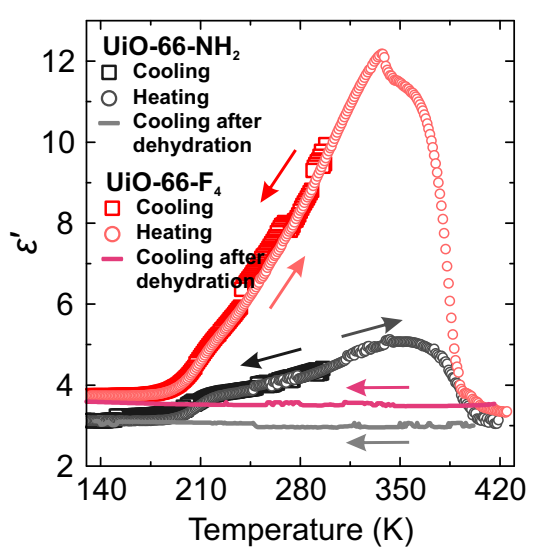

Figure 3. Real part of the complex dielectric permittivity of hydrated MOF samples measured at $1 \mathrm{MHz}$ frequency during the cooling-heating-cooling cycle.
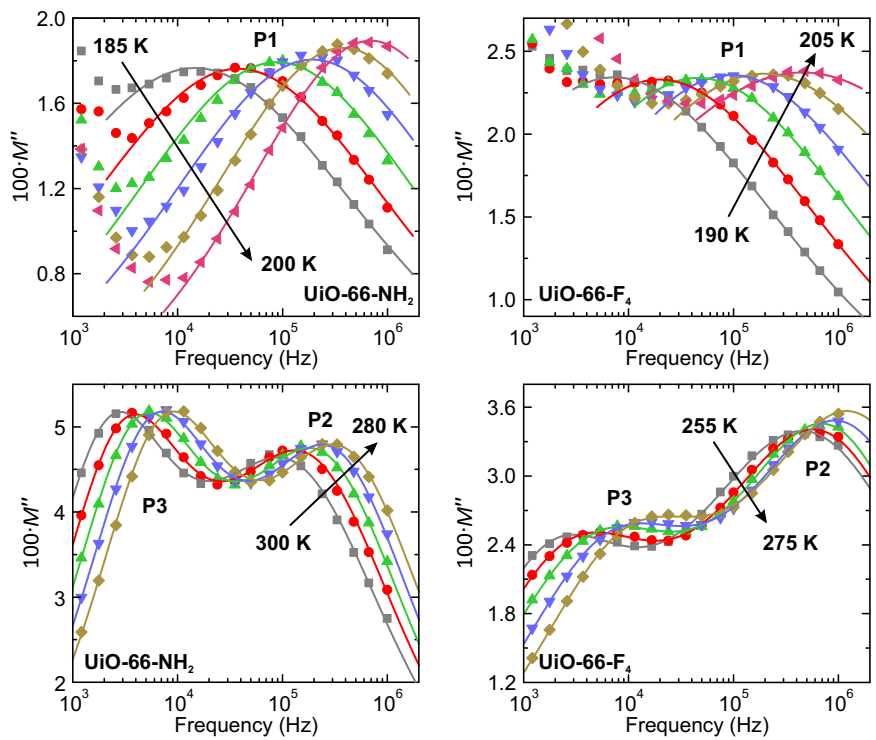

Figure 4. Frequency dependence of the imaginary part of the complex electric modulus of UiO-66- $\mathrm{NH}_{2}$ and $\mathrm{UiO}-66-\mathrm{F}_{4}$ hydrated MOFs presented at selected temperatures. Relaxation processes are indicated by the labels. Solid lines the best fits to the Cole-Cole relaxation processes.

The relaxation parameters of each process were extracted by fitting the obtained frequency dependences of $M^{\prime}$ and $M^{\prime \prime}$, where $\varepsilon^{*}$ in Equation (1) was expressed by a superposition of three independent Cole-Cole relaxations [36]:

$$
\varepsilon^{*}(\omega)=\varepsilon(\infty)+\sum_{n} \frac{\Delta \varepsilon_{n}}{1+\left(i \omega \tau_{n}\right)^{1-\alpha_{n}}} .
$$

Here, $\omega$ is the angular probing frequency, and $\varepsilon(\infty)$ is the dielectric permittivity in the infinite-frequency limit. The relaxations are described by different dielectric strengths $\Delta \varepsilon_{n}$ and mean relaxation times $\tau_{n}$ ( $n=1,2,3)$. The parameters $0<\alpha_{n} \leq 1$ determine the widths of the relaxations.

The obtained temperature dependences of the mean relaxation time for different processes are presented in Figure 5. The low temperature process P1 in both MOFs exhibits the Arrhenius-type temperature dependence of the mean relaxation time: $\tau=\tau_{0} \exp \left(E_{a} / k T\right)$, where $E_{a}$ and $\tau_{0}$ denote 
activation energy and attempt time, respectively, and $k$ is the Boltzmann constant. The determined activation energies for this process are 0.76 and $0.85 \mathrm{eV}$ for $\mathrm{UiO}-66-\mathrm{NH}_{2}$ and $\mathrm{UiO}-66-\mathrm{F}_{4}$, respectively (Table 1). Similar values of the activation energies were also reported for the same process in the unfunctionalized $\mathrm{UiO}-66$ and functionalized $\mathrm{UiO}-66-\mathrm{COOH}$ [33]. For both compounds, the relaxation strength $\Delta \varepsilon \approx 1.5$ and exponent $\alpha \approx 0.5$, which indicates a much broader dispersion than that of the Debye type. The broadening is likely caused by a superposition of more than one relaxation process with different characteristic relaxation times.

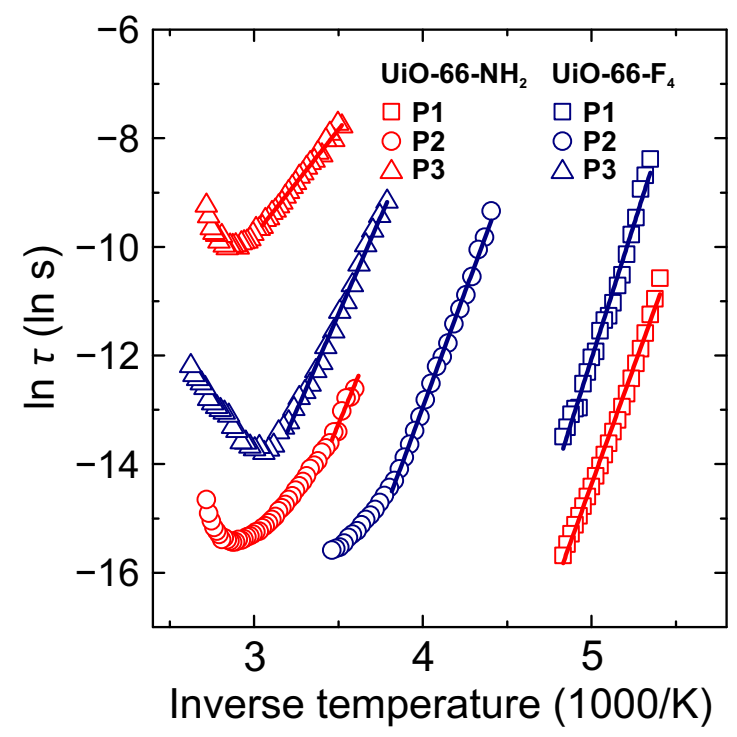

Figure 5. Inverse temperature dependence of the mean relaxation time of P1-P3 processes in hydrated UiO-66- $\mathrm{NH}_{2}$ and UiO-66- $\mathrm{F}_{4}$ MOFs. The lines are linear fits to the Arrhenius equation.

Table 1. Attempt time and activation energy for different processes in hydrated $\mathrm{UiO}-66-\mathrm{NH}_{2}$ and $\mathrm{UiO}-66-\mathrm{F}_{4}$ MOFs.

\begin{tabular}{ccc}
\hline \multicolumn{3}{c}{ UiO-66-NH ${ }_{2}$} \\
\hline Process & $\tau_{0}(\mathrm{~s})$ & $E_{a}(\mathrm{eV})$ \\
\hline P1 & $1.1(1) \times 10^{-25}$ & $0.76(2)$ \\
\hline P2 & $6.7(1) \times 10^{-18}$ & $0.65(1)$ \\
\hline P3 & $4.4(1) \times 10^{-10}$ & $0.34(1)$ \\
\hline & UiO-66-F \\
\hline Process & $\tau_{0}(\mathrm{~s})$ & $E_{a}(\mathrm{eV})$ \\
\hline P1 & $2.6(1) \times 10^{-27}$ & $0.85(2)$ \\
\hline P2 & $4.6(1) \times 10^{-21}$ & $0.73(1)$ \\
\hline P3 & $1.7(1) \times 10^{-16}$ & $0.62(1)$ \\
\hline
\end{tabular}

A similar response is widely observed for confined supercooled water in disordered porous materials such as silica hydrogels, Vycor glasses, molecular sieves, mineral clay, graphite oxide, cement-like materials and MOFs [28,39,40]. The majority of these materials are hydrophilic, generally because of the hydroxy groups on their surface and possess interconnected pore structures with a broad pore size distribution. In such systems, incomplete water filling of the pores is common and therefore water-surface interactions are promoted causing system-dependent alterations in the dynamical behavior of the confined water [39]. BDC linkers of the non-functionalized UiO-66 are hydrophobic, but the H-bond formation of the confined water molecules with hydroxy groups located at the inorganic nodes may be possible, similar to reported n-butane binding in these MOFs [41] and 
water molecules in the MIL-53 [42]. The functionalization of the BDC linker with polar $\mathrm{F}$ and $\mathrm{NH}_{2}$ groups introduces additional possible H-bonding sites in the framework resulting in the increased water-surface interactions. The determined activation energies for both compounds indicate that the dynamics of the confined water molecules are strongly hindered with considerably slower relaxation in UiO-66- $\mathrm{F}_{4}$ than in UiO-66- $\mathrm{NH}_{2}$ MOF.

A different temperature behavior is obtained for the mean relaxation time of $\mathrm{P} 2$ and $\mathrm{P} 3$ processes (Figure 5). Starting from the lower temperature, the mean relaxation time follows the Arrhenius law with increasing temperature. However, as temperature approaches the sample dehydration region, a clear minimum of $\ln \tau$ is observed indicating slowing down of the relaxation upon increase of temperature. High values of $\varepsilon^{\prime}$ at room temperature are commonly observed in various porous materials containing water $[26,28,43,44]$ and are usually interpreted by proton conductivity and /or Maxwell-Wagner polarization. Recent simulations of the proton transfer through the water clusters confined inside a nanometre sized cavities revealed that it proceeds via the Grotthuss diffusion and can even be enhanced, if the confinement is soft, allowing facile hydrogen-bond reorganization [45].

The obtained relaxation parameters of $\mathrm{P} 3$ process are strongly influenced by the amount of adsorbed water and different functional groups on the BDC linkers. The determined relaxation times for $\mathrm{P} 3$ process can be directly related to the DC conductivity for the charge transfer/relaxation process $\sigma_{D C} \sim \tau^{-1}$. Thus, the decrease of the relaxation time with temperature corresponds to the increase fo the DC conductivity with the same activation energy $E_{a}: \sigma T \sim \exp \left(-E_{a} / k T\right)$. The obtained $E_{a}$ value of $0.34 \mathrm{eV}$ for $\mathrm{P} 3$ process in hydrated UiO-66- $\mathrm{NH}_{2}$ is characteristic for Grotthuss conduction mechanism (typically $E_{a}<0.4 \mathrm{eV}$ ) [46,47].

In general, ionic conductivity can be expressed as $\sigma=Z e n \mu$, where $Z$ is the ionic charge, $e$ is the elementary charge, $n$ denotes the charge carrier concentration and $\mu$ is the carrier mobility. Therefore, functionalization of the UiO-66 framework can control proton conductivity by affecting the charge carrier concentration and/or mobility with improved conduction pathways at constant hydration levels. Relatively complicated structure of UiO-66 with interconnected octahedral and smaller tetrahedral pores may result in different intra-cage, inter-cage or window crossing mechanisms of proton transfer, similar as for a range of crystalline porous organic cages at high hydration levels [45]. High $E_{a}$ value for $\mathrm{P} 3$ process in UiO-66- $\mathrm{F}_{4}$ suggests that proton mobility could be also related to the pore and window size, which decreases after functionalization hindering the long range proton transfer. At temperature higher than $330 \mathrm{~K}$, where water desorption process starts dominating, water loss gradually breaks proton transfer pathways, which results in the observed slowing down of the relaxation time and lowering of the overall conductivity.

Interestingly, process $\mathrm{P} 2$ in both systems also displays qualitatively similar response to that of the process P3. Note that at lower temperatures, where process P2 enters our measurement frequency window, observation of linker rotation dynamics in functionalized UiO-66 MOFs was reported [30]. However, our dielectric measurements of the dehydrated samples (Figure 3) do not show any relaxation process due to the frequency dependent linker motion (at least in the sensitivity range of our measurement setup). In addition, process $\mathrm{P} 2$ in UiO-66-COOH MOF was ascribed to the dynamics of water/solid surface complex [33]. Similarity between the temperature dependences of the processes P2 and P3 indeed demonstrate that the origin of P2 process can be related to water conductivity response. In Grotthuss mechanism, the proton transfer through H-bonding network may be accompanied by a simultaneous sequential rotations of the molecules [48]. Ligand functionalization by polar groups can result not only in the creation of the additional charge carriers, but also in appearance of additional binding sites for possible $\mathrm{H}$-bond formation with adsorbed water molecules and development of new enhanced proton transfer routes.

In order to further elucidate the corresponding relaxation mechanisms, we performed dielectric measurements on UiO-66- $\mathrm{NH}_{2}$ and UiO-66- $\mathrm{F}_{4} \mathrm{MOF}$ samples with different adsorbed water content. After activation at about $420 \mathrm{~K}$, samples were cooled down to room temperature and left in the ambient atmosphere for $24 \mathrm{~h}$ to let natural water uptake. Afterwards, the same cooling-heating measurement 
cycle was performed. The obtained temperature and frequency dependences of the complex dielectric permittivity were compared with the ones recorded for highly hydrated samples. As can be seen from Figure 6a, only the low frequency process P3 was altered by insufficient hydration. Sample treatment in lower humidity atmosphere results in lower eps values, which is a clear indication of lower hydration level as reported for other porous compounds $[26,28,43,44]$.
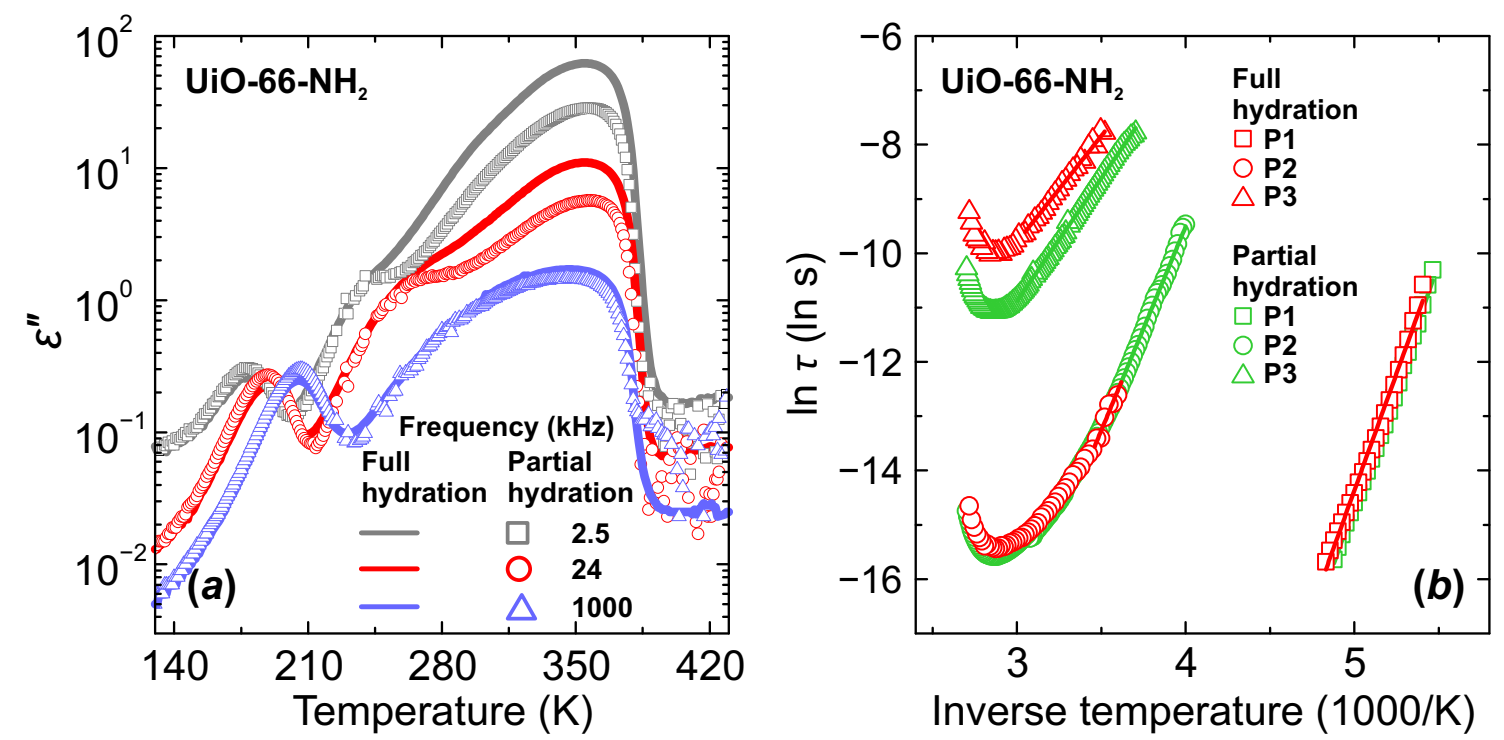

Figure 6. Temperature dependence of the (a) imaginary part of the complex dielectric permittivity and (b) mean relaxation time of fully and partially hydrated $\mathrm{UiO}-66-\mathrm{NH}_{2} \mathrm{MOF}$.

For P1 and P2 processes we observed no significant influence of the hydration level on their dielectric response or relaxation parameters, except that we were able to trace the P2 process to lower temperature due to less pronounced P3 process (Figure 6). These observations further imply that P1 and P2 processes could be related to water molecules near the pore surface. Our dielectric results suggest that at first the interfacial layers or sites are filled before formation of the proton transfer chain networks at higher hydration levels. Molecular dynamics simulation of microscopic mechanisms associated with the water-mediated proton transport in the MIL-53 MOF also show that at low water content water molecules tend to concentrate near the proton binding sites of the framework [42]. The observation that P2 has the same relaxation rate at different hydration levels (Figure 6b) suggests that this process can be related to water molecules that are situated close to the pore surface binding sites and form water/ligand H-bonded network for proton transfer. This process appears much earlier in the measured dielectric spectra with increasing temperature than $\mathrm{P} 3$ process, but has much higher activation energy (Table 1) in both $\mathrm{UiO}-66-\mathrm{NH}_{2}$ and $\mathrm{UiO}-66-\mathrm{F}_{4} \mathrm{MOFs}$, confirming stronger water-framework interaction influence.

When assessing the impact of linker modification on the dielectric permittivity data of adsorbed water, we must also stress that although $-\mathrm{F}$ group is considered much more hydrophobic, it still participates in the observed conduction and relaxation processes of confined water. Recent studies show that under certain circumstances, fluorine can act as a hydrogen bond acceptor [49]. Our results suggest that both $-\mathrm{NH}_{2}$ and $-\mathrm{F}$ groups influence the dynamics of the confined water molecules in qualitatively the same way by introducing additional binding sites for possible H-bond formation compared to unmodified UiO-66 MOF. The relatively weaker H-bond acceptor performance of $-\mathrm{F}$ results in slower relaxation of supercooled water molecules (process P1) and much higher activation energy for $\mathrm{P} 3$ proton transfer process in $\mathrm{UiO}-66-\mathrm{F}_{4}$, compared with $\mathrm{UiO}-66-\mathrm{NH}_{2}$, where all three water-MOF interactions are further enhanced because of a larger hydrophilicity of this group. 


\section{Materials and Methods}

\subsection{Sample Preparation}

UiO-66- $\mathrm{NH}_{2}$ : zirconyl chloride octahydrate $(21 \mathrm{mg})$ was dissolved in DMF $(3 \mathrm{~mL})$ at $50-60{ }^{\circ} \mathrm{C}$. This solution was added to a DMF solution $(1 \mathrm{~mL})$ in which 2-aminoterephthalic acid (54 mg) was dissolved completely. The combined mixture was homogenized by swirling before a small amount of glacial acetic acid $(0.98 \mathrm{~mL})$ was added. The resulting mixture was sonicated at $50-60{ }^{\circ} \mathrm{C}$ and placed in an oil bath $\left(120^{\circ} \mathrm{C}\right)$ under static condition for $24 \mathrm{~h}$. After cooling to room temperature, the precipitate was collected by centrifugation and washed with methanol three times. The isolated solid was then dried at room temperature under vacuum to give a yellow powder.

$\mathrm{UiO}-66-\mathrm{F}_{4}$ : tetrafluoroterephthalic acid $(30 \mathrm{mg})$ and zirconyl nitrate hydrate (45 $\left.\mathrm{mg}\right)$ were separately added into deionized water $(1.0 \mathrm{~mL}$ for each). After mixing these two solutions, a small amount of glacial acetic acid $(0.25 \mathrm{~mL})$ was added into the mixture. After stirring for $40 \mathrm{~h}$ at room temperature, the precipitate was collected by centrifugation. The as-synthesized sample was washed with D.I. water 3 times and then sequentially washed with ethanol 2 times, and finally immersed in acetone for 2 days. The isolated solid was then dried at room temperature under vacuum to give a white powder.

The scanning electron microscope (SEM) images (Figure S1 in the Supporting Information) revealed uniform size and shape of the crystallites. The average particle diameter is about 100 and $500 \mathrm{~nm}$ for $\mathrm{UiO}-66-\mathrm{NH}_{2}$ and $\mathrm{UiO}-66-\mathrm{F}_{4}$ samples, respectively. The obtained powder X-ray diffraction (PXRD) patterns of both synthesized compounds are typical for UiO-66 (see Figure S2).

\subsection{Dielectric Spectroscopy}

Dielectric spectroscopy measurements of UiO-66- $\mathrm{NH}_{2}$ and $\mathrm{UiO}-66-\mathrm{F}_{4}$ powders were performed in $1 \mathrm{kHz}-1 \mathrm{MHz}$ frequency and 130-430 $\mathrm{K}$ temperature ranges using a custom made cryostat and HP 4284A precision LCR meter. The sample was placed and slightly pressed using about $90 \mathrm{kPa}$ pressure in a cylindrical powder holder with top and bottom circular brass electrodes of 6 mm diameter. The typical sample thickness was $1.5 \mathrm{~mm}$. All experiments were performed at $1 \mathrm{~K} / \mathrm{min}$ cooling and heating rate. Measurements of the hydrated samples above room temperature were carried out in air, while a constant flow of nitrogen gas was used below this temperature and during the experiments with the dehydrated MOFs.

\section{Conclusions}

In summary, we investigated the dielectric response of functionalized $\mathrm{UiO}-66-\mathrm{NH}_{2}$ and $\mathrm{UiO}-66-\mathrm{F}_{4}$ MOFs. After hydration, both compounds exhibit a huge dielectric dispersion which can be separated into three partially overlapping processes. Sample dehydration causes disappearance of all these processes demonstrating that their origin is adsorbed water molecules. A detailed analysis of the mean relaxation times obtained from the frequency dependent data revealed that linker functionalization plays a significant role in affecting confined water cluster relaxation (P1 process) as well as the overall proton migration (processes P2 and P3). It is also plausible that linker functionalization can create new pathways for proton migration and potentially enhance the electrical conductivity. This observation is highly important for further application of $\mathrm{UiO}$ and similar funtionalized MOFs in electronic devices and sensors.

Supplementary Materials: The following are available online, Figure S1: SEM images of UiO-66- $\mathrm{NH}_{2}$ and UiO-66- $\mathrm{F}_{4}$ MOF crystallites, Figure S2: PXRD patterns of UiO-66- $\mathrm{NH}_{2}$ and UiO-66- $\mathrm{F}_{4} \mathrm{MOFs}$, Figure S3: Temperature dependence of the weight loss of hydrated UiO-66- $\mathrm{NH}_{2}$ and UiO-66- $\mathrm{F}_{4}$ MOFs, Figure S4: Frequency dependence of the complex dielectric permittivity of $\mathrm{UiO}-66-\mathrm{NH}_{2}$ hydrated MOF, Figure S5: Frequency dependence of the complex dielectric permittivity of $\mathrm{UiO}-66-\mathrm{F}_{4}$ hydrated MOF. 
Author Contributions: Conceptualization, S.B., M.Š. and M.K.; methodology, S.B., M.K., R.G. and J.B.; software, S.B.; validation, M.Š., R.G., M.K., K.C.-W.W., F.-K.S. and J.B.; formal analysis, S.B. and M.K.; investigation, S.B., J.-Y.Y., P.-C.H. and D.P.; resources, R.G. and J.B.; data curation, R.G.; writing-original draft preparation, M.Š.; writing-review and editing, M.Š.; visualization, S.B. and M.Š.; supervision, R.G., K.C.-W.W. and J.B.; project administration, R.G. and J.B.; funding acquisition, R.G. and J.B. All authors have read and agreed to the published version of the manuscript.

Funding: This research was funded by the Research Council of Lithuania (Project TAP LLT-4/2017).

Conflicts of Interest: The authors declare no conflict of interest.

\section{Abbreviations}

The following abbreviations are used in this manuscript:

MOF Metal-organic framework

SBU Secondary building unit

BDC 1,4-benzene-dicarboxylate

\section{References}

1. Meek, S.T.; Greathouse, J.A.; Allendorf, M.D. Metal-Organic Frameworks: A Rapidly Growing Class of Versatile Nanoporous Materials. Adv. Mater. 2011, 23, 249-267. [PubMed]

2. Kitagawa, S.; Kitaura, R.; Noro, S. Functional Porous Coordination Polymers. Angew. Chem. Int. Ed. 2004, 43, 2334-2375. [CrossRef]

3. Wu, H.; Gong, Q.; Olson, D.H.; Li, J. Commensurate Adsorption of Hydrocarbons and Alcohols in Microporous Metal Organic Frameworks. Chem. Rev. 2012, 112, 836-868. [CrossRef] [PubMed]

4. Sumida, K.; Rogow, D.L.; Mason, J.A.; McDonald, T.M.; Bloch, E.D.; Herm, Z.R.; Bae, T.H.; Long, J.R. Carbon Dioxide Capture in Metal-Organic Frameworks. Chem. Rev. 2012, 112, 724-781. [CrossRef] [PubMed]

5. Li, J.R.; Sculley, J.; Zhou, H.C. Metal-Organic Frameworks for Separations. Chem. Rev. 2012, 112, 869-932. [CrossRef]

6. Murray, L.J.; Dinca, M.; Long, J.R. Hydrogen Storage in Metal-Organic Frameworks. Chem. Soc. Rev. 2009, 38, 1294-1314. [CrossRef]

7. He, Y.; Zhou, W.; Qian, G.; Chen, B. Methane Storage in Metal-Organic Frameworks. Chem. Soc. Rev. 2014, 43, 5657-5678. [CrossRef] [PubMed]

8. Dhakshinamoorthy, A.; Li, Z.; Garcia, H. Catalysis and Photocatalysis by Metal Organic Frameworks. Chem. Soc. Rev. 2018, 47, 8134-8172. [CrossRef]

9. Wang, L.; Zheng, M.; Xie, Z. Nanoscale Metal-Organic Frameworks for Drug Delivery: A Conventional Platform with New Promise. J. Mater. Chem. B 2018, 6, 707-717. [CrossRef]

10. Kuppler, R.J.; Timmons, D.J.; Fang, Q.R.; Li, J.R.; Makal, T.A.; Young, M.D.; Yuan, D.; Zhao, D.; Zhuang, W.; Zhou, H.C. Potential Applications of Metal-Organic Frameworks. Coord. Chem. Rev. 2009, 253, 3042-3066. [CrossRef]

11. Cavka, J.H.; Jakobsen, S.; Olsbye, U.; Guillou, N.; Lamberti, C.; Bordiga, S.; Lillerud, K.P. A New Zirconium Inorganic Building Brick Forming Metal Organic Frameworks with Exceptional Stability. J. Am. Chem. Soc. 2008, 130, 13850-13851. [CrossRef] [PubMed]

12. Valenzano, L.; Civalleri, B.; Chavan, S.; Bordiga, S.; Nilsen, M.H.; Jakobsen, S.; Lillerud, K.P.; Lamberti, C. Disclosing the Complex Structure of UiO-66 Metal Organic Framework: A Synergic Combination of Experiment and Theory. Chem. Mater. 2011, 23, 1700-1718. [CrossRef]

13. Bristow, J.K.; Svane, K.L.; Tiana, D.; Skelton, J.M.; Gale, J.D.; Walsh, A. Free Energy of Ligand Removal in the Metal-Organic Framework UiO-66. J. Phys. Chem. C 2016, 120, 9276-9281. [CrossRef] [PubMed]

14. Kandiah, M.; Usseglio, S.; Svelle, S.; Olsbye, U.; Lillerud, K.P.; Tilset, M. Post-Synthetic Modification of the Metal-Organic Framework Compound UiO-66. J. Mater. Chem. 2010, 20, 9848-9851. [CrossRef]

15. Kandiah, M.; Nilsen, M.H.; Usseglio, S.; Jakobsen, S.; Olsbye, U.; Tilset, M.; Larabi, C.; Quadrelli, E.A.; Bonino, F.; Lillerud, K.P. Synthesis and Stability of Tagged UiO-66 Zr-MOFs. Chem. Mater. 2010, 22, 6632-6640. [CrossRef] 
16. Yang, Q.; Vaesen, S.; Ragon, F.; Wiersum, A.D.; Wu, D.; Lago, A.; Devic, T.; Martineau, C.; Taulelle, F.; Llewellyn, P.L.; et al. A Water Stable Metal-Organic Framework with Optimal Features for $\mathrm{CO}_{2}$ Capture. Angew. Chem. Int. Ed. 2013, 52, 10316-10320. [CrossRef]

17. Huang, Y.H.; Lo, W.S.; Kuo, Y.W.; Chen, W.J.; Lin, C.H.; Shieh, F.K. Green and Rapid Synthesis of Zirconium Metal-Organic Frameworks via Mechanochemistry: UiO-66 Analog Nanocrystals Obtained in One Hundred Seconds. Chem. Commun. 2017, 53, 5818-5821. [CrossRef]

18. Katz, M.J.; Moon, S.Y.; Mondloch, J.E.; Beyzavi, M.H.; Stephenson, C.J.; Hupp, J.T.; Farha, O.K. Exploiting Parameter Space in MOFs: A 20-Fold Enhancement of Phosphate-Ester Hydrolysis with UiO-66- $\mathrm{NH}_{2}$. Chem. Sci. 2015, 6, 2286-2291. [CrossRef]

19. Peterson, G.W.; McEntee, M.; Harris, C.R.; Klevitch, A.D.; Fountain, A.W.; Soliz, J.R.; Balboa, A.; Hauser, A.J. Detection of an Explosive Simulant via Electrical Impedance Spectroscopy Utilizing the UiO-66- $\mathrm{NH}_{2}$ Metal-Organic Framework. Dalton Trans. 2016, 45, 17113-17116. [CrossRef]

20. Yot, P.G.; Yang, K.; Ragon, F.; Dmitriev, V.; Devic, T.; Horcajada, P.; Serre, C.; Maurin, G. Exploration of the Mechanical Behavior of Metal Organic Frameworks UiO-66(Zr) and MIL-125(Ti) and their $\mathrm{NH}_{2}$ Functionalized Versions. Dalton Trans. 2016, 45, 4283-4288. [CrossRef]

21. Yang, Q.; Wiersum, A.D.; Llewellyn, P.L.; Guillerm, V.; Serre, C.; Maurin, G. Functionalizing Porous Zirconium Terephthalate UiO-66(Zr) for Natural Gas Upgrading: A Computational Exploration. Chem. Commun. 2011, 47, 9603-9605. [CrossRef]

22. Jasuja, H.; Zang, J.; Sholl, D.S.; Walton, K.S. Rational Tuning of Water Vapor and $\mathrm{CO}_{2}$ Adsorption in Highly Stable Zr-Based MOFs. J. Phys. Chem. C 2012, 116, 23526-23532. [CrossRef]

23. Kolokolov, D.I.; Stepanov, A.G.; Jobic, H. Mobility of the 2-Methylimidazolate Linkers in ZIF-8 Probed by ${ }^{2}$ H NMR: Saloon Doors for the Guests. J. Phys. Chem. C 2015, 119, 27512-27520. [CrossRef]

24. Khudozhitkov, A.E.; Kolokolov, D.I.; Stepanov, A.G. Characterization of Fast Restricted Librations of Terephthalate Linkers in MOF UiO-66(Zr) by ${ }^{2} \mathrm{H}$ NMR Spin-Lattice Relaxation Analysis. J. Phys. Chem. C 2018, 122, 12956-12962. [CrossRef]

25. Kolokolov, D.I.; Maryasov, A.G.; Ollivier, J.; Freude, D.; Haase, J.; Stepanov, A.G.; Jobic, H. Uncovering the Rotation and Translational Mobility of Benzene Confined in UiO-66 (Zr) Metal-Organic Framework by the ${ }^{2}$ H NMR-QENS Experimental Toolbox. J. Phys. Chem. C 2017, 121, 2844-2857. [CrossRef]

26. Bermudez-Garcia, J.M.; Vicent-Luna, J.M.; Yanez-Vilar, S.; Hamad, S.; Sanchez-Andujar, M.; Castro-Garcia, S.; Calero, S.; Senaris-Rodriguez, M.A. Liquid Self-Diffusion of $\mathrm{H}_{2} \mathrm{O}$ and DMF Molecules in Co-MOF-74: Molecular Dynamics Simulations and Dielectric Spectroscopy Studies. Phys. Chem. Chem. Phys. 2016, 18, 19605-19612. [CrossRef]

27. Knebel, A.; Geppert, B.; Volgmann, K.; Kolokolov, D.I.; Stepanov, A.G.; Twiefel, J.; Heitjans, P.; Volkmer, D.; Caro, J. Defibrillation of Soft Porous Metal-Organic Frameworks with Electric Fields. Science 2017, 358, 347-351. [CrossRef]

28. Balciunas, S.; Simenas, M.; Pavlovaite, D.; Kinka, M.; Shieh, F.K.; Wu, K.C.W.; Banys, J.; Grigalaitis, R. Low-Frequency Dipolar Dynamics and Atmospheric Effects in ZIF-90 Metal-Organic Framework. J. Phys. Chem. C 2019, 123, 631-636. [CrossRef]

29. Gonzalez-Nelson, A.; Coudert, F.X.; van der Veen, M.A. Rotational Dynamics of Linkers in Metal-Organic Frameworks. Nanomaterials 2019, 9. [CrossRef]

30. Devautour-Vinot, S.; Maurin, G.; Serre, C.; Horcajada, P.; Paula da Cunha, D.; Guillerm, V.; de Souza Costa, E.; Taulelle, F.; Martineau, C. Structure and Dynamics of the Functionalized MOF Type UiO-66(Zr): NMR and Dielectric Relaxation Spectroscopies Coupled with DFT Calculations. Chem. Mater. 2012, 24, 2168-2177. [CrossRef]

31. Devautour-Vinot, S.; Martineau, C.; Diaby, S.; Ben-Yahia, M.; Miller, S.; Serre, C.; Horcajada, P.; Cunha, D.; Taulelle, F.; Maurin, G. Caffeine Confinement into a Series of Functionalized Porous Zirconium MOFs: A Joint Experimental/Modeling Exploration. J. Phys. Chem. C 2013, 117, 11694-11704. [CrossRef]

32. Devautour-Vinot, S.; Diaby, S.; da Cunha, D.; Serre, C.; Horcajada, P.; Maurin, G. Ligand Dynamics of Drug-Loaded Microporous Zirconium Terephthalates-Based Metal-Organic Frameworks: Impact of the Nature and Concentration of the Guest. J. Phys. Chem. C 2014, 118, 1983-1989. [CrossRef]

33. Planchais, A.; Devautour-Vinot, S.; Salles, F.; Ragon, F.; Devic, T.; Serre, C.; Maurin, G. A Joint Experimental/ Computational Exploration of the Dynamics of Confined Water/Zr-Based MOFs Systems. J. Phys. Chem. C 2014, 118, 14441-14448. [CrossRef] 
34. El-Mehalmey, W.A.; Ibrahim, A.H.; Abugable, A.A.; Hassan, M.H.; Haikal, R.R.; Karakalos, S.G.; Zaki, O.; Alkordi, M.H. Metal-Organic Framework@Silica as a Stationary Phase Sorbent for Rapid and Cost-Effective Removal of Hexavalent Chromium. J. Mater. Chem. A 2018, 6, 2742-2751. [CrossRef]

35. Kim, H.; Yang, S.; Rao, S.R.; Narayanan, S.; Kapustin, E.A.; Furukawa, H.; Umans, A.S.; Yaghi, O.M.; Wang, E.N. Water Harvesting from Air with Metal-Organic Frameworks Powered by Natural Sunlight. Science 2017, 356, 430-434. [CrossRef]

36. Schonhals, A.; Kremer, F. Broadband Dielectric Spectroscopy, 1st ed.; Springer: Berlin/Heidelberg, Germany, 2003.

37. Sieradzki, A.; Pawlus, S.; Tripathy, S.N.; Gagor, A.; Ciupa, A.; Mączka, M.; Paluch, M. Dielectric Relaxation Behavior in Antiferroelectric Metal Organic Framework $\left[\left(\mathrm{CH}_{3}\right)_{2} \mathrm{NH}_{2}\right]\left[\mathrm{FeIIIFeII}(\mathrm{HCOO})_{6}\right]$ Single Crystals. Phys. Chem. Chem. Phys. 2016, 18, 8462-8467. [CrossRef]

38. Hodge, I.; Ngai, K.; Moynihan, C. Comments on the Electric Modulus Function. J. Non-Cryst. Solids 2005, 351, 104-115. [CrossRef]

39. Cerveny, S.; Mallamace, F.; Swenson, J.; Vogel, M.; Xu, L. Confined Water as Model of Supercooled Water. Chem. Rev. 2016, 116, 7608-7625. [CrossRef]

40. Bergman, R.; Swenson, J. Dynamics of Supercooled Water in Confined Geometry. Nature 2000, 403, $283-286$. [CrossRef]

41. Sharp, C.H.; Abelard, J.; Plonka, A.M.; Guo, W.; Hill, C.L.; Morris, J.R. Alkane-OH Hydrogen Bond Formation and Diffusion Energetics of n-Butane within UiO-66. J. Phys. Chem. C 2017, 121, 8902-8906. [CrossRef]

42. Paesani, F. Molecular Mechanisms of Water-Mediated Proton Transport in MIL-53 Meta-Organic Frameworks. J. Phys. Chem. C 2013, 117, 19508-19516. [CrossRef]

43. Sanchez-Andujar, M.; Yanez-Vilar, S.; Pato-Doldan, B.; Gomez-Aguirre, C.; Castro-Garcia, S.; Senaris-Rodriguez, M.A. Apparent Colossal Dielectric Constants in Nanoporous Metal Organic Frameworks. J. Phys. Chem. C 2012, 116, 13026-13032. [CrossRef]

44. Xue, C.; Yao, Z.Y.; Liu, S.X.; Luo, H.B.; Zou, Y.; Li, L.; Ren, X.M. Dielectric Anomaly and Relaxation Natures in a Zn-Cr Pillar-Layered Metal-Organic Framework with Cages and Channels. J. Solid State Chem. 2017, 250, 107-113. [CrossRef]

45. Liu, M.; Chen, L.; Lewis, S.; Chong, S.Y.; Little, M.A.; Hasell, T.; Aldous, I.M.; Brown, C.M.; Smith, M.W.; Morrison, C.A.; et al. Three-Dimensional Protonic Conductivity in Porous Organic Cage Solids. Nat. Commun. 2016, 7, 12750. [CrossRef] [PubMed]

46. Ramaswamy, P.; Wong, N.E.; Shimizu, G.K.H. MOFs as Proton Conductors-Challenges and Opportunities. Chem. Soc. Rev. 2014, 43, 5913-5932. [CrossRef]

47. Lim, D.W.; Sadakiyo, M.; Kitagawa, H. Proton Transfer in Hydrogen-Bonded Degenerate Systems of Water and Ammonia in Metal-Organic Frameworks. Chem. Sci. 2019, 10, 16-33. [CrossRef] [PubMed]

48. Marx, D. Proton Transfer 200 Years after von Grotthuss: Insights from Ab Initio Simulations. ChemPhysChem 2006, 7, 1848-1870. [CrossRef] [PubMed]

49. Schneider, H.J. Hydrogen Bonds with Fluorine. Studies in Solution, in Gas Phase and by Computations, Conflicting Conclusions from Crystallographic Analyses. Chem. Sci. 2012, 3, 1381-1394. [CrossRef]

(C) 2020 by the authors. Licensee MDPI, Basel, Switzerland. This article is an open access article distributed under the terms and conditions of the Creative Commons Attribution (CC BY) license (http://creativecommons.org/licenses/by/4.0/). 